\title{
Automatic and Attentional Components in Perception of Shape-at-a-Slant
}

\author{
William Epstein and Barbara E. Lovitts \\ University of Wisconsin-Madison
}

\begin{abstract}
In perceiving shape-at-a-slant it is assumed that a sequence of operations is executed. The aim of these experiments was to determine the extent to which execution of these operations requires allocation of attention. Three hypotheses were considered: zero automaticity-that all of the operations require attention; partial automaticity - that the operations culminating in a representation of projective shape and slant-in-depth are automatic while the combinatorial operations culminating in a distally correlated shape require attention; full automaticity - that the entire sequence of operations is automatic, proceeding without allocation of attention. To decide among these hypotheses, subjects performed forced-choice shape recognition tests under two conditions: In the shape-directed condition subjects were motivated to process shape. In the numerosity-directed condition subjects were motivated to direct attention to discrimination of numerosity, thereby causing attention to be diverted from processing of shape. Examination of the pattern of choices on the recognition test showed results that conformed best to the hypothesis of partial automaticity.
\end{abstract}

When an object is presented at different orientations or when the same object is viewed from different vantage points, the object presents correspondingly different aspects. For example, a circle presents a variety of elliptical shapes when it occupies various positions other than the frontal parallel plane. These variations are little reflected in perception that tends to remain constant and relatively true to the distal shape.

According to a long-held view (contemporary versions are offered by Epstein, 1973; Epstein \& Hatfield, 1978; Epstein, Hatfield, \& Muise, 1977; Rock, 1975, 1977, 1983, among others), invariance of perceived shape and the close correspondence between perceived shape and distal shape are mediated by a computation-like process that acts on information about projective shape and on information about the position of the object

This research was conducted as part of Barbara $E$. Lovitts's master's thesis at the University of Wisconsin.

We are indebted to Irvin Rock, who functioned as action editor for this article.

The advice of Arthur Glenberg, Lola Lopes, and Gregg Oden is also gratefully acknowledged.

Requests for reprints should be sent to William Epstein, University of Wisconsin, W. J. Brogden Psychology Building, Madison, Wisconsin 53706. relative to the observer to generate a description of shape that is constant and distally correlated.

One instantiation of this form of account is illustrated in Figure 1. The visual system is supposed to possess neuronal structures that are responsive to the properties of optical input that carry information about the projective shape and position in space of the object. Registration of this information culminates in a description or representation of projective shape and orientation in depth. Notice that in this instantiation the descriptions of projective shape and of orientation in depth are constructed in parallel and that the descriptions are independent at this stage of the process. Presumably, if the process were arrested at this point and if the products were accessible to conscious report, an observer would report a proximally correlated shape and an orientation in depth as close to the objective orientation as the information in stimulation allows. In the succeeding stage, the observer computes a value for objective shape, based on the descriptions of projective shape and orientation in depth. Application of the computational rule or algorithm guarantees a distally correlated solution when the representations of projective shape and orientation in depth are adequate. 


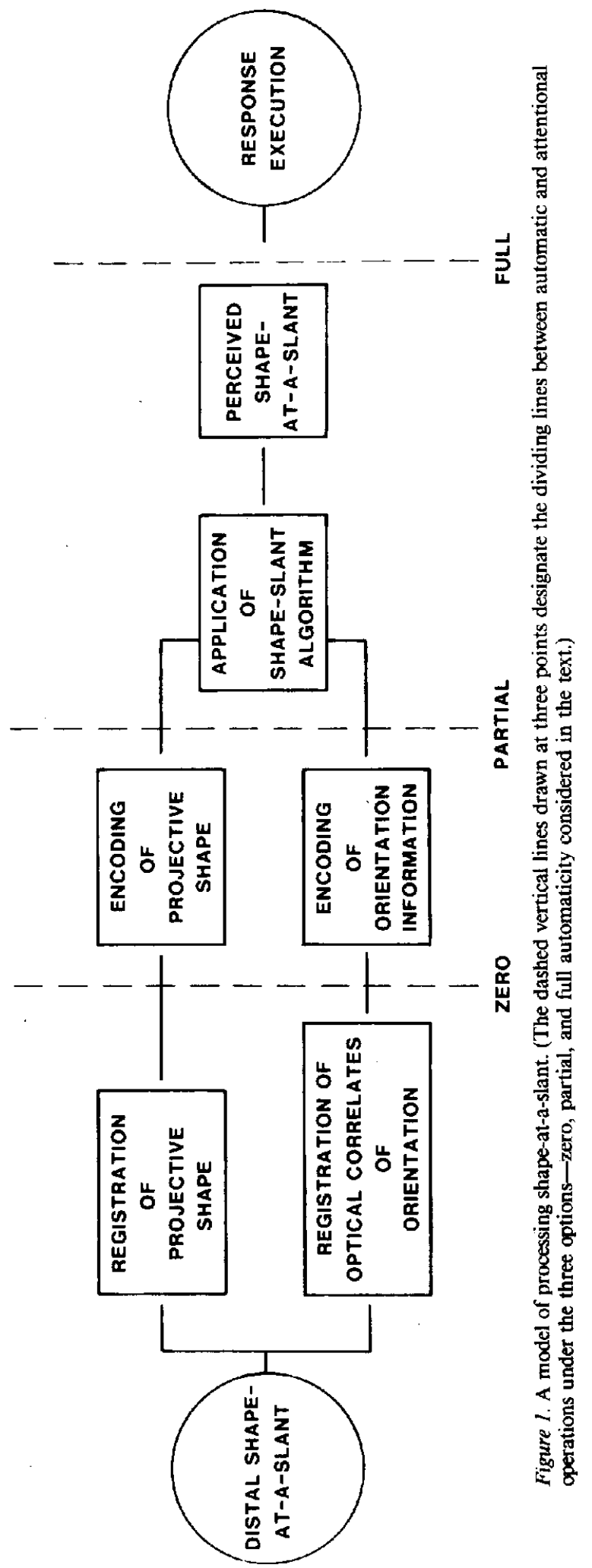


The aim of the present experiments was to examine the involvement of attention in the process of perceiving shape-at-a-slant. ${ }^{1}$ Three possibilities may be delineated, differing in the degree in which the process is assumed to depend on allocation of attention: (a) The full-automaticity option proposes that the entire process runs off automatically, that none of the operations is dependent on allocation of attention. (b) The zero-automaticity option assumes that all of the component operations require allocation of attention. (c) The partial-automaticity option stipulates that a number of the operations require allocation of attention, whereas other operations are automatic.

More must be specified about the partialautomaticity option to make it amenable to test. In particular we must specify which operations are expected to be automatic and which operations require attention. One plausible dividing line is indicated in Figure 1. The operations leading to the representation of projective shape and orientation in depth are assumed to be automatic; application of the computational rule for derivation of a distally correlated shape is assumed to demand attention. ${ }^{2}$ Drawing the line at this point is recommended partly by empirical considerations and partly by expedience. The empirical considerations are the findings of Epstein et al. (1977) and Leibowitz and Bourne (1956) concerning the effect of abbreviated exposure on perceived shape. Leibowitz and Bourne found that when a shape that is rotated in depth is presented for exposure durations of $100 \mathrm{~ms}$ or less, the subject reports a shape corresponding to the projective shape. Epstein et al. (1977) confirmed this result in an experiment that deployed backward masking to control processing time. If we assume that the rapid automatic operations elude masking and the slower attentiondemanding operations are preempted by the mask, we have justification for drawing the dividing line as we have done. The second reason for drawing the line at this point is that this decision generates a number of predictions that help decide among the three options.

The general plan of both experiments is to compare reports of perceived shape obtained under two conditions, one that directs atten- tion to processing shape-at-a-slant and the other that withdraws attention from processing shape. The three options set out above have distinctive expectations concerning reported shape when attention has been withdrawn from processing of shape.

The full automaticity option predicts that reported shape, say, the shape selected on a forced-choice test, will be independent of allocation of attention. If the prevailing conditions, for example, available depth information, favor distally correlated choices when attention is directed to processing shape, the same distally correlated choices are expected when attention is withdrawn from shape processing.

The zero-automaticity option predicts that when attention is withdrawn from processing shape, the choice of a matching shape should be a random selection from the alternatives made available to the subject. Inasmuch as all operations demand attention, withdrawal of attention will mean that neither a representation of projective shape nor a representation of distally correlated shape will be formed. If forced to choose, the observer's choices will bear no systematic relation to either projective or objective shape.

The partial-automaticity option, in the version offered above, predicts that when attention is withdrawn from processing shape, the selected shape should correspond most closely to the projective shape. If the subject is offered a choice between two or three alternatives, the subject should choose the alter-

\footnotetext{
I The literature concerning automaticity and attention is extraordinarily rich conceptually and empirically. Among the empirical studies, those by Goldstein and Fink (1981), Rock and Gutman (1981), and Rock, Schauer, and Halper (1976) showing no effect of inattention on perception of two-dimensional drawings come closest to our question. However, there are no published works of which we are aware that have investigated the processing of shapes in three-dimensional space. For this reason, we can offer nothing in the way of directly relevant history. Nevertheless, it will be obvious throughout that in formulating the question and in designing the experimental attack we have drawn heavily from contemporary investigators of attention.

2 The partial automaticity option has a family resemblance to Treisman's (Treisman \& Gelade, 1980; Treisman \& Schmidt, 1982) feature integration theory of attention. Treisman supposes that features are automatically encoded but that formation of an integrated perceptual object requires allocation of attention.
} 
native that reproduces the projective shape of the target, even when an objective match for the target is included among the set of alternatives. This expectation follows from the decision to treat as automatic all of the operations prior to the combination of projective shape and orientation information. As a consequence, the subject has available a description of projective shape but no distally correlated description unless attention is allocated to computation of a distally correlated description.

In summary, we propose to look to the pattern of choices obtained under the two attentional conditions for evidence that will help us decide among the three options. All options predict that distally correlated choices will predominate when attention is directed to processing of shape. The options lead to differing predictions when attention is withdrawn from processing of shape.

\section{Experiment 1}

The chief experimental manipulation was designed to control allocation of attention to the processing of shape while assuring that the optical input is the same under both attentional conditions. The latter requirement was satisfied by presenting the same set of shapes under conditions that encouraged the subjects to fixate on the shapes even when the task did not require processing of shape. Each of eight shapes was presented individually. Arrayed vertically along the vertical axis of rotation on the surface of each shape were a number of dots. The subjects in the shape-directed condition were instructed that after a predetermined series of trials (each pass through all eight shapes constituting a trial), they would be asked to select matches for the standard shapes. The subjects in the numerosity-directed condition were instructed that the task was to discriminate between arrays containing odd and even numbers of dots. No mention was made of shape discrimination. By arranging the dots along the central axis of the shape, we guaranteed that the shapes would be imaged foveally. Immediately following the last trial a three-alternative forced-choice shape recognition test was administered to all subjects.

\section{Method}

Subjects. Fifty members of the university community-undergraduates, graduate students, and employees-participated in the first run of Experiment 1 as paid volunteers. A different group of 50 unpaid volunteers, drawn entirely from the student body in an introductory psychology class, were the subjects in a second run of Experiment 1.

Stimuli. The standard shapes were selected from Vanderplas and Garvin's (1959) compilation of irregularly contoured shapes. For each shape Vanderplas and Garvin presented an association value and a measure of complexity based on Attneave and Arnoult's (1956) procedure. As standards we selected 8 eight-point and 8 four-point shapes. Half of the shapes at each level of complexity had high association value $(M=48 \%)$, half had low association value $(M=29 \%)$. Association value and complexity were crossed to create four classes of standards each containing four shapes. Two shapes from each set of four served as standards in Experiment $1 \mathrm{~A}$; the remaining two shapes in each set were used in Experiment 1B. Two replicas of each of the eight shapes were cut from white posterboard. Each of these standards was mounted on a stalk that was aligned with the vertical axis of the standard. The stalks were painted flat black; they were not visible against the black background of the viewing chambers. For the distances at which the standard was viewed, the standards subtended horizontal visual angles ranging from $3.35^{\circ}$ to $6.93^{\circ}$ and vertical visual angles ranging from $6.41^{\circ}$ to $8.14^{\circ}$. A vertical array of black dots, 5-8 in number, was affixed along the vertical axis of each shape. The dots (commercially produced Mecanorma CS 220) were $3 \mathrm{~mm}$ in diameter and were spaced at intervals of $3 \mathrm{~mm}$. One member of each pair of duplicate standards exhibited an odd number of dots; the other exhibited an even number of dots. All of the standards were rotated in depth around the vertical axis by $60^{\circ}$ when presented for viewing. The viewing distance was $65 \mathrm{~cm}$.

The test shapes were miniature versions of the standards. The horizontal visual angles of the test shapes ranged from $0.56^{\circ}$ to $2.81^{\circ}$; the vertical visual angles ranged from $1.68^{\circ}$ to $2.92^{\circ}$. The test shapes were presented in the tachistoscope in sets of three. Each set included one miniature replica of the objective shape of the standard (alternative $\mathrm{O}$ ), one miniature replica of projective shape of the standard at $60^{\circ}$ (alternative $P$ ), and one miniature foil (alternative F). For half of the test sets the foil was $4 \mathrm{~mm}$ wider $\left(F_{w}\right)$ than the objective miniature; for half of the test sets the foil was $4 \mathrm{~mm}$ narrower $\left(F_{N}\right)$ than the projective miniature. Two sets of test shapes are shown in Figure 2. The presence of foils insured that neither the objective match nor the projective match occupied a fixed position (widest, narrowest, respectively) in the test set. Because for half of the test trials, taken over subjects, each standard was accompanied by a foil that was narrower than the projective equivalent and on half the trials a foil that was wider than the objective match, the objective match was widest and intermediate in width equally often, and the projective match was narrowest and intermediate equally often.

All of the miniatures were cut from white paper and affixed in sets of three to large sheets of black posterboard. 
The three shapes comprising a set were arranged in a single column as shown in Figure 2. Dots were not included on the test shapes. Two sets of eight test cards were prepared so that the spatial positions of the alternatives within the set for a given standard were not the same for all subjects. In addition, the spatial positions of the alternatives on the test cards were randomized over the eight standards. Each test card was presented individually in the frontal plane. From the subject's vantage point, the test display appeared as three miniature white forms arranged in a column in uniformly dark surrounding.

Apparatus. The main components of apparatus were a two-field tachistoscope modified for presentation of shapes rotated in depth, a microprocessor that controlled the tachistoscope and other aspects of the procedure such as order of presentation, a three-button response panel, and a printer that recorded the subject's responses. Each field in the tachistoscope contained a carousel with eight stalks bearing shapes positioned at equal intervals around the perimeter of the carousel. The stalks were fixed in position so that when a standard shape was centered in the viewing field, the shape was oriented at $60^{\circ}$ with respect to the subject's frontal parallel plane. When test cards replaced the standards, the cards always were oriented in the frontal parallel plane.

Procedure. The subjects were assigned in random order to the two attentional conditions. Throughout the experiment, all of the subjects viewed standard and test shapes binocularly. Subjects assigned to the shape-directed condition were instructed that the task of the experiment involved shape discrimination. The nature of the threealternative forced-choice test was described. The subjects were advised that the altematives comprising each set would be similar, but exact information about the makeup of the three-alternative set was not provided. As illustration, a single randomly contoured curvilinear shape was

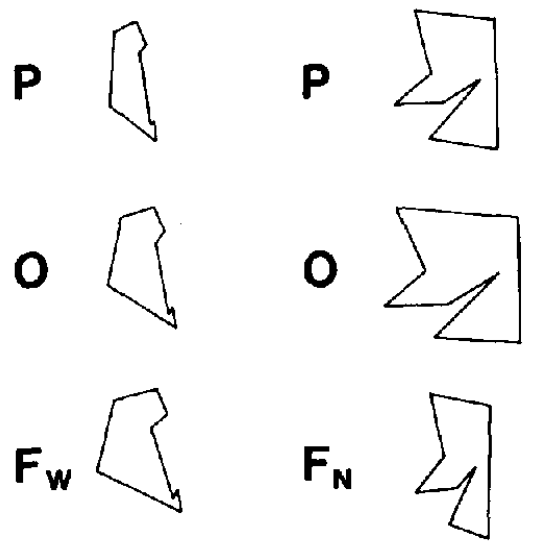

Figure 2. Examples of the sets of test shapes used for two of the standard shapes in Experiment 1. (O is the objective match. $P$ is the projective match. $F_{N}$ in the right-hand set is the foil narrower than $P ; F_{W}$ in the lefthand set is the foil wider than $O$. Spatial position [top, bottom, intermediate] of the alternatives within each set of test shapes was varied between subjects.) shown in the frontal parallel plane, followed by an appropriately designed test card, and the experimenter indicated the correct choice. The subjects were instructed to ignore the dots; the presence of the dots was attributed to the requirements of a different experiment. Administration of the instructions was followed by 20 viewing trials. At each trial each of the eight shapes was presented once. The order of presentation of the shapes was randomized from trial to trial. The exposure duration for each shape was $1 \mathrm{~s}$.; the inverval between presentations averaged $5 \mathrm{~s}$. At the conclusion of these viewing trials, the eight test cards were presented one at a time in random order. The subjects were informed that each test set included an exact replica of one of the shapes observed during the viewing trials. The task was "to determine which of the three miniature shapes comes closest to matching the shape you observed during the viewing trials." Subjects indicated a choice by pressing one of the three response buttons. Testing was self-paced although subjects were encouraged not to perseverate.

The subjects in the numerosity-directed condition were instructed that the task of the experiment involved an odd-even discrimination. The nature of the stimuli was described, and the irregularly contoured curvilinear shape was exhibited as an example. For this stage of the experiment, one of the three response buttons was concealed, leaving one button marked odd and the other marked even. The subject was instructed to respond odd or even as speedily after onset of the standard as was consistent with high accuracy. A numerosity discrimination was secured for each presentation of a standard. The subjects were advised to ignore the shapes. The presence of the shapes was attributed to the requirements of a different experiment. Following the instructions, the standards were presented. Each subject in the numerositydirected condition was yoked to a subject in the shapedirected condition so that the pairs of yoked subjects were exposed to the standards in the same order. At the conclusion of the 20 viewing trials, the shape-matching test was introduced. The information about the test and the instructions were the same as those provided to the shape-directed subjects.

Experiment 1 was run twice. The runs differed in two respects: (a) As noted in the section describing the standard forms, two different sets of eight standards, matched for complexity and association value, were prepared. One set served as standards in the original run (Experiment IA); the other set served as standards in the second run (Experiment 1B). Our purpose was to sample a larger number of shapes. (b) The second run was conducted by a different experimenter, (the second author), who at the time of data collection did not know the outcome of the first run nor did she know the theoretical framework of the study.

\section{Results}

The results for each run of the experiment will be reported separately under the headings Experiment $1 \mathrm{~A}$ and $1 \mathrm{~B}$.

Experiment 1A. The left side of Table 1 shows the distribution of choices among the 
Table 1

Percentage of Choices in Each Category for the Two Altentional Conditions

\begin{tabular}{|c|c|c|c|c|c|c|}
\hline \multirow{2}{*}{$\begin{array}{l}\text { Attentional } \\
\text { condition }\end{array}$} & \multicolumn{3}{|c|}{$\begin{array}{c}\text { Experiment } \\
1_{\mathrm{A}}\end{array}$} & \multicolumn{3}{|c|}{$\begin{array}{c}\text { Experiment } \\
\text { 1B }\end{array}$} \\
\hline & 0 & $\mathbf{P}$ & $\mathbf{F}$ & $\mathrm{O}$ & $\mathbf{P}$ & $\mathrm{F}$ \\
\hline Shape-directed & 55 & 28 & 18 & 61 & 24 & 17 \\
\hline Numerosity-directed & 31 & 47 & 22 & 42 & 31 & 28 \\
\hline
\end{tabular}

Note. $\mathrm{O}$ designates the test alternatives that matched the objective shapes of the standards; $P$ designates the alternatives that matched the projective shapes of the standards; and $F$ designates the test alternatives that serve as foils.

three types of alternatives for the two attentional conditions. The objective alternative (O) was most frequently chosen when attention was directed to processing of shape, whereas the projective alternative $(P)$ was most frequently chose when attention was directed to numerosity discrimination. The foil (F) was chosen with about equal frequency under the two attentional conditions.

Examination of the individual subject data provided corroboration. In the shape-directed condition, $80 \%$ of the subjects selected more Os than Ps, and $20 \%$ of the subjects selected more Ps than Os. In the numerosity-directed condition, $60 \%$ of the subjects selected more Ps than Os; $20 \%$ selected more Os than Ps; and $20 \%$ selected an even number of Os and Ps.

The two attentional conditions were compared for each response ( $\mathrm{O}, \mathrm{P}$, and $\mathrm{F}$ ) category. Separate analyses of variance showed that the difference between the attentional conditions was highly significant for both the $\mathrm{O}$ and $\mathrm{P}$ categories, $F(1,48)=28.66, p<.001$, and $F(1,48)=15.78, p<.001$, respectively. The attentional conditions did not differ significantly with respect to the number of choices of the foil, $F(1,48)=1.17, p>.05$. An Attentional Condition $\times$ Response Category interaction for $\mathrm{O}$ and $\mathrm{P}$ is clearly discernible in the left half of Table 1. In a separate analysis, omitting the $F$ category, this interaction was highly significant, $F(1,48)=25.32$, $p<.001$.

The average percent correct for the numerosity discrimination task under the numerosity-directed condition was $79.7 \%$. This level of performance is evidence that the subjects were allocating processing resources to the task.

Experiment 1B. The right side of Table 1 shows the distribution of choices among the three types of alternatives for the two attentional conditions. The results for the shapedirected condition resembled the results for this condition in the first run. Alternative $O$ was chosen on $61 \%$ of the test trials. Examination of the individual subject data showed that $88 \%$ of the shape-directed subjects selected more Os than Ps. The results for the numerosity-directed condition differed from the results of Experiment $1 \mathrm{~A}$. Although there was a sharp decline in the proportion of $\mathrm{O}$ responses compared to the proportion obtained under the shape-directed condition, the $\mathbf{P}$ response was not dominant. Examination of individual subject data showed that only $32 \%$ of the numerosity-directed subjects selected more Ps than Os, and 52\% actually selected more Os than Ps.

Separate analyses of variance showed that the differences between the attentional conditions were highly significant for response category $O, F(1,48)=12.74, p<.001$, and response category $\mathrm{F}, F(1,48)=5.59, p<.05$. The difference between the attentional conditions for response category $\mathbf{P}$ was not significant, $F(1,48)=2.05, p>.05$. However, the separate analysis to assess the interaction between attentional conditions and response category, which is clearly discernible in the right half of Table 1, was significant, $F(1$, $48)=7.27, p<.01$. In corroboration of Experiment $1 \mathrm{~A}$, the preference for $\mathrm{O}$ over $\mathrm{P}$ was significantly greater under the shapedirected condition than under the numerositydirected condition.

The average percent correct for the numerosity discrimination task under the numerosity-directed condition was $80.6 \%$.

Influence of foil width. The data were reexamined to determine what effect the foil may have had on test performance. In Table 2 the data are conditionalized on the presence of $F_{W}$ and $F_{N}$ in the test set averaged over the two runs. No clear pattern is apparent. The only consistency was that choices of the foil were more likely to occur when $F_{W}$ was offered rather than $F_{N}$.

Conclusion. The results of Experiment 1 showed that direction of attention significantly 
Table 2

Percentage of Responses in Each Category Conditionalized on Foil Width

\begin{tabular}{cccccc}
\hline & \multicolumn{3}{c}{ Attentional condition } \\
\cline { 2 - 3 } & \multicolumn{2}{c}{$\begin{array}{c}\text { Shape- } \\
\text { directed }\end{array}$} & & \multicolumn{2}{c}{$\begin{array}{c}\text { Numerosity- } \\
\text { directed }\end{array}$} \\
\cline { 2 - 3 } \cline { 5 - 6 } Response & $F_{W}$ & $F_{N}$ & & $F_{W}$ & $F_{N}$ \\
\hline$O$ & 43 & 57 & & 56 & 43 \\
$P$ & 50 & 50 & & 38 & 62 \\
$F$ & 78 & 22 & & 60 & 40 \\
\hline
\end{tabular}

Note. $\mathrm{O}, \mathrm{P}$, and $\mathrm{F}$ designate the objective and projective matches and the foil, respectively. $F_{W}$ was wider than the objective test alternative; $F_{N}$ was narrower than the projective test alternative.

affected the subjects' test performance. The proportion of choices of objectively identical shapes as matches was significantly greater under the shape-directed condition. This outcome is not compatible with the full-automaticity hypothesis, which would expect selection of matches to be independent of direction of attention. A decision between the zero- and partial-automaticity options cannot be reached on the basis of Experiment 1. As set out earlier, this decision rests not only on a reduction in the proportion of $\mathrm{O}$ choices under the numerosity-directed condition but also on the division of responses between response alternatives $P$ and $F$. The zeroautomaticity hypothesis requires an even distribution among the three alternatives; the partial-automaticity hypothesis requires that the $\mathbf{P}$ alternative be dominant. The results did not conform clearly to either pattern.

\section{Experiment 2}

Experiment 2 was a modified version of Experiment 1. The aim of the modifications was to eliminate a number of features of Experiment 1 that in retrospect seemed undesirable. The following changes were introduced: (a) The instructions to the numerositydirected group were edited to eliminate any references that may have suggested that shape should be an object of attention. In addition, presentation of the amoeboid familiarization shape was deleted for both groups. (b) The number of viewing trials was reduced from 20 to 10 . An exploratory study showed that halving the number of trials did not affect performance on the shape recognition test when subjects were directed to process shape. By reducing the number of viewing trials we hoped to lessen the likelihood that subjects habituating to the demands of numerosity discrimination in the numerosity-directed condition might direct attention to shape. (c) The three-alternative forced-choice test was replaced by a two-alternative forced-choice test (2AFC). The composition of the $2 \mathrm{AFC}$ test allowed us to search for a pattern of converging results in seeking to decide among the three options and also allowed for an assessment of the effects of attentional allocation in the absence of the distracting presence of a foil. The 2AFC test was administered in test booklets that presented pairs of miniature shapes, each pair on a separate page. (d) Only the 8 eight-point shapes from Experiments $1 \mathrm{~A}$ and $1 \mathrm{~B}$ were used. Although the four-point and eight-point shapes were matched in average association value, we thought it less likely that spontaneous verbal labeling would occur with the more complex eight-point shapes.

\section{Method}

Subjects. The subjects were 50 University of Wisconsin undergraduates enrolled in an introductory psychology course. The subjects were assigned in random order to the two experimental conditions.

Composition of test. The test booklet was composed of 32 sheets, each presenting two miniature shapes side by side. Sixteen of the test trials paired an $O$ and a $P$ alternative with each standard shape tested twice. These 16 trials were supplemented by four trials of each of the following four types of pairs: $\mathrm{O}-\mathrm{F}_{\mathrm{W}}, \mathrm{P}-\mathrm{F}_{\mathrm{N}}, \mathrm{P}-\mathrm{F}_{\mathrm{W}}$, and $O-F_{N}$. The assignment of four shapes to each of these four test categories was varied between subjects so that each shape was subjected to all four test types. The order of test trials was randomized for each subject.

Procedure. The procedure resembled Experiment 1 with the exceptions noted above. The test was administered immediately following the 10th viewing trial and was self-paced. The subject indicated a choice by marking one of the two alternatives on each trial.

\section{Results}

Table 3 sets out three patterns of results for the five types of test trials. When the subject is directed to process shape, it is expected that whenever a test pair includes an objective shape, whether the objective shape is paired with a projective shape or a 
Table 3

Expected Outcomes for Two-Alternative Forced-Choice Tests for the Various Automaticity Hypotheses

\begin{tabular}{lcccc}
\hline & \multicolumn{4}{c}{ Attentional condition } \\
\cline { 3 - 5 } & & \multicolumn{3}{c}{ Numerosity-directed } \\
\cline { 3 - 5 } Test & Shape- & Degree of automaticity \\
\cline { 3 - 5 } pair & directed & Full & Partial & Zero \\
\hline O-P & O $>$ P & O $>$ P & $O<P$ & $O=P$ \\
O-F & $O>F_{W}$ & $O>F_{W}$ & $O=F_{W}$ & $O=F_{W}$ \\
O-F & $O>F_{N}$ & $O>F_{N}$ & $O=F_{N}$ & $O=F_{N}$ \\
$P-F_{W}$ & $P=F_{W}$ & $P=F_{W}$ & $P>F_{W}$ & $P=F_{W}$ \\
$P-F_{N}$ & $P=F_{N}$ & $P=F_{N}$ & $P>F_{N}$ & $P=F_{N}$ \\
\hline
\end{tabular}

Note. $\mathrm{O}$ designates the objective test alternative; $\mathrm{P}$ designates the projective alternative; $F_{W}$ and $F_{N}$ designate the wide and narrow foils, respectively.

foil, the subject will choose the objective shape. However, when the test pair does not offer an objective match, that is, when $P$ and $F$ make up the test pair, the subject will divide the choices equally between $\mathrm{P}$ and $\mathrm{F}$. This expectation rests on the assumption that a representation of $P$ is not readily accessible once the entire sequence of operations culminating in a distally correlated perceived shape has been completed. Consequently, a $\mathrm{P}-\mathrm{F}$ pair does not offer a match, and the choices divide between the two unacceptable alternatives. $^{3}$

Turning now to the numerosity-directed subjects, Table 3 shows that if processing shape-at-a-slant is fully automatic, then the pattern of results for the five test types will be identical to the pattern for the shapedirected subjects. But if, as Experiment 1 has given us cause to believe, either partial- or zero-automaticity prevails, the pattern of results for the numerosity-directed condition will differ from the shape-directed condition. If none of the operations are automatic and if attention has been completely withdrawn from processing of shape, the choices should be divided equally between the two alternatives on each test type.

Yet another outcome is expected on the partial-automaticity hypothesis. Consulting Table 3, we note that for each test type the two attentional conditions differ. The differences all flow from the following underlying premises. For the shape-directed subject a representation of $O$ is available at the time of test, and a representation of $P$ is not accessible; for the numerosity-directed subject the reverse is true, that is, a representation of $P$ is available, a representation of $O$ is not. Consequently, if the test pair includes a replica of $P$, the numerosity-directed subject has a clear choice, and $P$ will be favored even if it is paired with $O$. However, if $P$ is not a member of the pair, then no match has been offered for the current representation, and random choices ensue.

Table 4 presents the distribution of responses for each test type for the two attentional conditions. On test type $\mathrm{O}-\mathrm{P}$ there is evidence of a strong interaction: The $\mathrm{O}$ alternative was favored under the shape-directed condition, whereas the $\mathbf{P}$ alternative was favored under the numerosity-directed condition. As Table 3 shows, this outcome is compatible only with the partial-automaticity option. The evidence from the $\mathrm{O}-\mathrm{F}_{\mathrm{W}}$ test trials is equivocal; neither the shape-directed nor the numerositydirected subjects exhibited a significant preference. Turning to the $\mathrm{O}-\mathrm{F}_{\mathrm{N}}$ trials, we find additional evidence of partial automaticity: The shape-directed subjects showed a strong preference $(80 \%)$ for $\mathrm{O}$, whereas the numerosity-directed subjects divided their choices about evenly between $O(53 \%)$ and $F(47 \%)$. The outcome for test type $P-F_{W}$ also fits well with the partial-automaticity option: The shape-directed subjects divided their choice between $P$ and $F_{W}$, whereas the numerositydirected subjects selected $\mathrm{P}$ more frequently $(57 \%)$ than $F_{W}(42 \%)$. The outcome for the $\mathrm{P}-\mathrm{F}_{\mathrm{N}}$ test trials is theoretically equivocal although the numerosity-directed subjects exhibited a strong preference for $P$ over $F_{N}$

\footnotetext{
${ }^{3}$ The claim that percipients have access to a proximally correlated and a distally correlated description has been urged by Mack (1978), who distinguishes among "modes" of perceiving: the proximal mode and the constancy mode. In Rock's (1977) development of a neo-Helmholtzian theory, the proximal mode is realized even when perceptual constancy is obtained, that is, even when distally correlated perception is attained. Consequently, Rock might challenge the assumption that the proximally correlated description is unaccessible when the full sequence of operations has been completed. If the proximally correlated descriptions remain available, then when the subject is offered a choice between $P$ and F, P should be preferred.
} 
Table 4

Percentage of Choices Under the Shape-Directed and Numerosity-Directed Conditions for the Five Types of Test Trials in Experiment 2

\begin{tabular}{ccc}
\hline & \multicolumn{2}{c}{ Attentional condition } \\
\cline { 2 - 3 } Test & Shape-directed & $\begin{array}{c}\text { Numerosity- } \\
\text { directed }\end{array}$ \\
pair & $\mathrm{O}=68-\mathrm{P}=32$ & $\mathrm{O}=46-\mathrm{P}=54$ \\
\hline $\mathrm{O}-\mathrm{P}$ & $\mathrm{O}=47-\mathrm{F}_{\mathrm{W}}=53$ & $\mathrm{O}=52-\mathrm{F}_{\mathrm{W}}=48$ \\
$\mathrm{O}-\mathrm{F}_{\mathrm{W}}$ & $\mathrm{O}=80-\mathrm{F}_{\mathrm{N}}=20$ & $\mathrm{O}=47-\mathrm{F}_{\mathrm{N}}=53$ \\
$\mathrm{O}-\mathrm{F}_{\mathrm{N}}$ & $\mathrm{P}=44-\mathrm{F}_{\mathrm{W}}=56$ & $\mathrm{P}=58-\mathrm{F}_{\mathrm{W}}=42$ \\
$\mathrm{P}-\mathrm{F}_{\mathrm{W}}$ & $\mathrm{P}=76-\mathrm{F}_{\mathrm{N}}=24$ & $\mathrm{P}=64-\mathrm{F}_{\mathrm{N}}=36$ \\
\hline
\end{tabular}

Note. Over all subjects in each attentional condition there was a grand total of 400 test responses for test pair O-P and 100 test responses for each of the remaining four test types. $O$ designates the objective test alternative; $P$ the projective alternative; $F_{W}$ and $F_{N}$ the wide and narrow foils, respectively.

(64\% vs. $36 \%)$; the shape-directed subjects exhibited an even stronger preference in the same direction. Table 5 presents the results of the individual analyses of variance testing the differences between the number of choices of the two alternatives comprising each test type for the two attentional conditions.

The average percent correct on the numerosity discrimination task for the numerositydirected subjects was $78.9 \%$. As in Experiment 1 , there is evidence that the subjects were processing the numerosity task.

\section{Supplementary Experiment}

The average percent correct on the numerosity-directed task was $79.7 \%, 80.6 \%$, and $78.9 \%$ for Experiments $1 \mathrm{~A}, 1 \mathrm{~B}$, and 2, respectively. Although this level of performance is clear evidence of processing of the dot displays, the fact that performance was less than perfect may seem to indicate that the subjects in the numerosity-directed condition were dividing attention between the demands of numerosity discrimination and processing of other aspects of the display, perhaps shape. In order to assess this possibility, 25 subjects were tested for numerosity discrimination in the absence of shapes or other features that might elicit allocation of processing resources. These 25 subjects received the same sequence of odd and even dot displays as did the first 25 subjects in Experiment 2 . The only differ- ence was that the dots were placed on white field-filling placards rather than on shapes. Consequently, for these subjects the only features that might elicit processing were the arrays of dots. If the fact that numerosity discrimination was limited to $80 \%$ correct in the preceding experiments was due to the allocation of a portion of processing resources to shape, then performance in the absence of shapes or any other identifiable distractors should improve. On the other hand, if performance represents limitations on the quality of the data, for example, limits of resolution or any other factor inherent in the numerositydiscrimination task itself, then performance should not differ from the earlier levels. The average percent correct in this supplementary experiment was $77.6 \%$, which does not differ significantly from the average of $78.9 \%$ for the earlier experiments. There was no support for the suspicion that the less than perfect performance on the numerosity discrimination reflects a division of attention between processing of numerosity and processing of shape.

\section{General Discussion}

Of the three options set out in the introduction, the partial-automaticity hypothesis has survived the experimental tests best. The evidence of significant effects of direction of attention on the frequency of selection of the $O$ and $P$ alternatives is not readily accommodated by either the full-automaticity or

Table 5

Results of Analyses of Variance of Experiment 2

\begin{tabular}{lrcccc}
\hline & \multicolumn{3}{c}{ Attentional condition } \\
\cline { 2 - 3 } & \multicolumn{1}{c}{ Shape-directed } & & \multicolumn{2}{c}{ Numerosity-directed } \\
\cline { 2 - 3 } \cline { 5 - 6 } pest & $F(1,48)$ & $p$ & $F(1,48)$ & $p$ \\
\hline pair & O-P & 95.12 & $<.001$ & 1.91 & $n s$ \\
O-F & 0.93 & $n s$ & 0.56 & $n s$ \\
O-F & 73.55 & $<.001$ & 0.70 & $n s$ \\
P-F & 1.85 & $n s$ & 4.48 & $<.05$ \\
P-F & 44.82 & $<.001$ & 13.36 & $<.001$ \\
\hline
\end{tabular}

Note. $\mathrm{O}, \mathrm{P}$, and $\mathrm{F}$ designate the objective and projective matches and the foil, respectively: $F_{W}$ was wider than the objective test alternative. $F_{N}$ was narrower than the projective test alternative. 
the zero-automaticity hypothesis. On the other hand, despite a number of discrepancies, the data conformed reasonably well to the expectations of our version of partial automaticity. Keeping in mind that the orienting task is likely to fail to achieve its purpose on some trials, that is, that on some trials numerositydirected subjects may process shape, the overall pattern of the data constitutes a plausible basis for building the case for partial autonomy.

The fact that our conclusions are based on the outcome of a test for recognition memory deserves comment. Conventionally, examinations of the perception of shape-at-a-slant have used procedures that make no demands on long-term memory and minimal, if any, demands on short-term memory. Memory is bypassed by the simple expedient of presenting the standard to be judged and a comparison set or an adjustable variable shape available for simultaneous inspection or for repeated alternating inspections. Our procedure, which administered a test after repeated viewings and in the absence of the standards, may seem cause for concern. By so plainly involving memory, the test performance may seem to say more about memory for shape than perception of shape. Nevertheless, without denying that retrieval and comparison operations affected performance on the forced-choice test, it is difficult to formulate an account of our results that draws exclusively on memory operations. The challenge to a memory account is to explain the pattern of choices, a pattern that conforms in a principled way to the partial-automaticity hypothesis. It is not apparent that such an account can be developed without adopting prior assumptions about the representations of the standards in memory. In turn, these representations must significantly reflect the output of initial perceptual encoding.

The experimental procedure did not exercise control over eye movements. We cannot assert that contour scanning, if scanning did occur, occurred under both conditions. Given the different tasks assigned under the shapedirected and numerosity-directed conditions, it may be plausibly argued that although scanning may have occurred under the former condition, scanning is unlikely under the latter condition. We believe that two consid- erations mitigate the concern with differential scanning: (a) The preferred response under the numerosity-directed condition was the projective shape. If it is supposed that scanning did not occur under this condition, then we may infer that scanning is not necessary for registration and description of the projective shape of foveated distal shapes. The evidence of accurate perception of form in the absence of the opportunity to scan contoured shapes (for a review see Rock, 1983, Ch. 3, 4, 7) is consistent with this supposition. Because all that the hypothesized scanning under the shape-directed condition might contribute is registration of projective shape, if such registration can be accomplished in the absence of scanning, differences in scanning between the two conditions seem unlikely to affect perceived shape. (b) A review of the literature offered by Stark and Ellis (1981) concludes that the relation between scanpaths and pattern perception or pattern recognition has not been established even when subjects are allowed ample time, for example, $30 \mathrm{~s}$, for scanning.

At this point we might note a difference in the nature of the evidence generated by this experiment and the evidence provided by the experiments reported by Rock et al. (Rock \& Gutman, 1981; Rock, Schauer, \& Halper, 1976) and Goldstein and Fink (1981). These latter investigators looked for evidence of the contribution of attention to form perception (using drawings of shapes) by comparing recognition memory for unattended and attended forms that had been presented simultaneously for foveal inspection. Evidence that allocation of attention is necessary for form perception and recognition was deduced from the finding that only attended forms were recognized with high probability, whereas unattended forms were correctly identified infrequently. In fact, in Rock and Gutman's (1981) study, forms not previously presented during the inspection period and unattended previously presented forms were incorrectly designated as "old" (false positives) with equal frequency. So in these experiments inattention is accompanied by poor performance, that is, failure to discriminate old from new. The reliance on general deficit to support the claims for the importance of attention encourages the concern that the 
differences between the attended and unattended forms might be due to poorer memory for unattended forms rather than to differences in perception. (Rock \& Gutman, 1981, and Rock et al., 1976, offer plausible arguments for setting aside the concern.) In contrast, the present experimental paradigm is not a search for general decline in correct recognition when attention is withdrawn. It is true that subjects in this condition had no reason to intend to learn or remember the figures, and thus one might argue that they would have been poorer in recognition and likely to base many choices on mere guessing. But in our opinion, it is inappropriate to characterize performance under the numerosity-directed condition as poorer than performance under the shape-directed condition. The difference between the two conditions reflects the differences in the representations of shape at different stages in the processing sequence. Instead of general deficit, our paradigm looks to specific differences derived in a principled way from a model of perceiving shape-at-a-slant. As we contended above, this aspect of our approach helps reduce its vulnerability to an account stressing differences in memory for form under the two attentional conditions.

The evidence of the present experiments suggests that the model offered in Figure 1 is incomplete because it takes no account of the contribution of attention. The present results show that the description generated by the perceptual process will vary, depending on whether attention is engaged. Only when attention is engaged will the process culminate in a distally correlated shape. This assertion finds no corroboration in the everyday experience of ordinary seeing. Except under unusual circumstances, the percipient is not conscious of an effort to perceive shape-at-aslant. Moreover, no case of ordinary seeing comes to mind to suggest that computation of shape-at-a-slant is other than mandatory. The absence of awareness and the mandatory character of a process are often taken as features that identify an automatic process. Nevertheless, although the phenomenology cannot be denied, there is no necessary inconsistency between granting these points and asserting that the combinatorial operation is resource demanding. Precedent for discon- necting these putative attributes of automaticity can be found in the experiments by Paap and Ogden (1981) and by Regan (1981) concerning letter encoding and by Kahneman and Chajczyk (1983) in a study of dilution of Stroop effects. Paap and Ogden (1981) showed that the activation of memory codes for letters is obligatory and not under conscious control but that letter encoding is not cost free, that is, encoding demands processing resources, as evidenced in interference with a secondary task. Kahneman and Chajczyk (1983) reported that the reading of color words in the Stroop task is vulnerable to interference from other objects in the visual field. Reading in the Stroop task, although not subject to voluntary control, nevertheless, does require allocation of processing resources. In agreement with Paap and Ogden (1981) and Regan (1981), Kahneman and Chajczyk (1983) conclude that "the two criteria of automaticity-lack of voluntary control and absence of attention demands-are both conceptually and empirically independent" (p. 507).

\section{References}

Attneave, F., \& Arnoult, M. D. (1956). The quantitative study of shape and pattern perception. Psychological Bulletin, 53, 452-471.

Epstein, W. (1973). The process of "taking-into-account" in visual perception. Perception, 2, 267-285.

Epstein, W., \& Hatfield, G. (1978). Functional equivalence of masking and cue reduction in perception of shape at a slant. Perception \& Psychophysics, 23, 137-144.

Epstein, W., Hatfield, G., \& Muise, G. (1977). Perceived shape at a slant as a function of processing time and processing load. Journal of Experimental Psychology: Human Perception and Performance, 3, 473-483.

Goldstein, E. B., \& Fink, S. I. (1981). Selective attention in vision: Recognition memory for superimposed line drawings. Journal of Experimental Psychology: Human Perception and Performance, 7, 954-967.

Kahneman, D., \& Chajczyk, D. (1983). Tests of the automaticity of reading: Dilution of Stroop effects by color-irrelevant stimuli. Journal of Experimental Psychology: Human Perception and Performance, 9, 497509.

Leibowitz, H., \& Bourne, L. (1956). Time and intensity as determiners of perceived shape. Journal of Experimental Psychology, 51, 277-281.

Mack, A. (1978). Three modes of visual perception. In H. I. Pick, Jr., \& E. Saltzman (Eds.), Modes of perceiving and processing information (pp. 171-186). Hillsdale, NJ: Erlbaum.

Paap, K. R., \& Ogden, W. G. (1981). Letter encoding is an obligatory but capacity-demanding operation. Jour- 
nal of Experimental Psychology: Human Perception and Performance, 7, 518-528.

Regan, J. (1981). Automaticity and learning: Effect of familiarity on naming letters. Journal of Experimental Psychology: Human Perception and Performance, 7, 180-195.

Rock, 1. (1975). An introduction to perception. New York: Macmillan.

Rock, I. (1977). In defense of unconscious inference. In W. Epstein (Ed.), Stability and constancy in visual perception (pp. 321-374). New York: Wiley.

Rock, I. (1983). The logic of perception. Boston: Bradford Press.

Rock, I., \& Gutman (1981). The effect of inattention on form perception. Journal of Experimental Psychology: Human Perception and Performance, 7, 275-285.

Rock, I., Schauer, R., \& Halper, F. (1976). Form perception without attention. Quarterly Journal of Experimental Psychology, 28, 429-440.
Stark, L., \& Ellis, S. R. (1981). Scanpaths revisited: Cognitive models direct active looking. In D. F. Fisher, R. A. Monty \& J. W. Senders (Eds.), Eye movements: Cognition and visual perception (pp. 193-226). Hillsdale, NJ: Erlbaum.

Treisman, A., \& Gelade, G. (1980). A feature-integration theory of attention. Cognitive Psychology, 12, 97-136.

Treisman, A., \& Schmidt, H. (1982). Hlusory conjunctions in the perception of objects. Cognitive Psychology, 14, 107-141.

Vanderplas, J. M., \& Garvin, E. A. (1959). The association value of random shapes. Joumal of Experimental Psychology, 57, 147-154.

Received June 18, 1984 Revision received January 4, 1985 